\title{
Dual chemical reaction networks and implications for Lyapunov-based structural stability
}

\author{
Franco Blanchini $^{a}$ and Giulia Giordano ${ }^{b}$
}

\begin{abstract}
Given a class of (bio)Chemical Reaction Networks (CRNs) identified by a stoichiometric matrix $S$, we define as dual reaction network, $\mathrm{CRN}^{*}$, the class of (bio)Chemical Reaction Networks identified by the transpose stoichiometric matrix $S^{\top}$. We consider both the dynamical systems describing the time evolution of the species concentrations and of the reaction rates. First, based on the analysis of the Jacobian matrix, we show that the structural (i.e., parameter-independent) local stability properties are equivalent for a CRN and its dual CRN*. We also assess the structural global stability properties of the two dual networks, analysing both concentration and rate representations. We prove that the existence of a polyhedral (or piecewise-linear) Lyapunov function in concentrations for a CRN is equivalent to the existence of a piecewise-linear in rates Lyapunov function for the dual $\mathbf{C R N}^{*}$; in fact, if $V$ is a polyhedral Lyapunov function for a CRN, the dual polyhedral function $V^{*}$ is a piecewise-linear in rates Lyapunov function for the dual network. We finally show how duality can be exploited to gain additional insight into biochemical reaction networks.
\end{abstract}

Index Terms-Biological systems; Systems biology; Lyapunov methods

\section{INTRODUCTION}

$\mathbf{W}$ E adopt a duality perspective to perform the structural stability analysis of (bio)chemical reaction networks [6], [14], [16], [18], [19], [20]. A property is structural if it is satisfied by all the systems belonging to a class, characterised by a structure, regardless of parameter values [10] (as opposed to robust properties, which only need to be preserved under large parameter variations). The structural analysis of Chemical Reaction Networks (CRNs) [18], [19], [20] has provided fundamental results, such as the celebrated zero-deficiency theorem and one-deficiency theorem [16] and a lot of subsequent work [5], [13], [21]. In particular, structural stability is investigated in [7], [8], [9] for a wide class of (bio)chemical reaction networks, under the sole requirement of monotonic reaction rates; the nonlinear system equations are absorbed into a linear differential inclusion and then the piecewise-linear, i.e., polyhedral [11], Lyapunov function (if any) certifying the structural stability of the class of systems is computed through an iterative algorithm. The complementary class of piecewise-linear in rates Lyapunov functions has been proposed in [1], [2], [3], [4] for the stability analysis of chemical reaction networks.

Here, we propose the concept of dual chemical reaction network. Given a chemical reaction network with stoichiometric matrix $S \in \mathbb{Z}^{n \times m}$, involving $n$ species and $m$ reactions,

\footnotetext{
${ }^{a}$ Dipartimento di Matematica, Informatica e Fisica, Università degli Studi di Udine, Italy. blanchini@uniud.it

${ }^{a}$ Dipartimento di Ingegneria Industriale, Università degli Studi di Trento, Italy. giulia.giordanodunitn. it
}

its dual network has stoichiometric matrix $S^{\top} \in \mathbb{Z}^{m \times n}$, and thus involves $m$ species and $n$ reactions. We credit Famili and Palsson for proposing the compound map, associated with $-S^{\top}$ [15]; however, the equivalence stability results we obtain for the dual network, associated with $S^{\top}$, cannot be obtained for the compound map in [15].

Our main results establish the equivalence between the structural stability properties of a CRN and its dual CRN*:

- a CRN is structurally locally stable within its stoichiometric compatibility class (S-LSSC) if and only if its dual $\mathrm{CRN}^{*}$ is S-LSSC;

- the existence of a piecewise-linear Lyapunov function (PLF) for a CRN is equivalent to the existence of a piecewise-linear in rates Lyapunov function (PLF in rates) for its dual $\mathrm{CRN}^{*}$;

- in particular, if $V$ is a PLF for a CRN, then the dual function $V^{*}$ is a PLF in rates for its dual CRN*.

Concluding examples show the application of duality results to the structural stability analysis of biochemical networks.

\section{DuAl CRNs And DuAl StRuctures}

We consider general (bio)chemical reaction networks corresponding to nonlinear systems of the form

$$
\dot{y}=S \tilde{g}(y)+g_{0},
$$

where $y \in \mathbb{R}_{+}^{n}$ is the state vector of species concentrations, $\tilde{g}(y) \in \mathbb{R}_{+}^{m}$ is the vector of reaction rate functions and $g_{0} \geq 0$ is a vector of constant influxes; $S \in \mathbb{Z}^{n \times m}$ is the stoichiometric matrix, whose $S_{i j}$ entry represents the net amount of the $i$ th species produced or consumed by the $j$ th reaction, excluding the contribution of constant influxes. Therefore, $S_{i j}<0$ if the $i$ th species is involved in the $j$ th reaction as a reagent, while $S_{i j}>0$ if the $i$ th species is a product of the $j$ th reaction.

We assume there exists an equilibrium $\bar{y}$ such that $S \tilde{g}(\bar{y})+$ $g_{0}=0$. Without loss of generality, we can therefore consider the shifted system with state $x=y-\bar{y}$ and dynamics

$$
\dot{x}(t)=S[\tilde{g}(x(t)+\bar{y})-\tilde{g}(\bar{y})] \doteq S g(x(t)) .
$$

Then, to analyse the stability of the equilibrium, without restriction we will consider a model of the form

$$
\dot{x}(t)=S g(x(t)),
$$

with no constant influxes $g_{0}$, and its equilibrium at zero.

Hence, we make the following standing assumptions.

Assumption 1: Function $g(0)=0$, hence system (2) admits an equilibrium $\bar{x}=0$. 
Remark 1: Since we consider the shifted system around the equilibrium, now set at zero, there is no need to assume that $x$ and $g(x)$ are componentwise non-negative.

Assumption 2: All the component functions of vector $g(x)$ are continuously differentiable.

Assumption 3: The reaction rate function $g_{j}(\cdot)$ depends on $x_{i}$ if and only if $S_{i j}<0$, and $g_{j}=0$ when $x_{i}=0$

The coexistence of Assumptions 1 and 3 requires that, if there are no negative entries in the $j$ th column of $S$, then $g_{j} \equiv 0$.

Assumption 3 rules out autocatalytic reactions, such as $A \rightarrow$ $2 A$ or $A+B \rightarrow 2 B$.

Assumption 4: If function $g_{j}(\cdot)$ depends on $x_{i}$, then $\partial g_{j} / \partial x_{i}>0$.

Definition 1: Given a CRN of the form (2), its dual CRN* is the system

$$
\dot{z}(t)=S^{\top} h(z(t)),
$$

where function $h$ satisfies the same assumptions as $g$, but for the new stoichiometric matrix $S^{*}=S^{\top}$.

If a CRN has $n$ species and $m$ reactions, then its dual $\mathrm{CRN}^{*}$ has $m$ species and $n$ reactions.

Uppercase letters denote chemical species and the corresponding lowercase letters denote their concentrations; reaction rate functions are also denoted by lowercase letters.

Example 1: Consider the chemical reaction network CRN shown in Fig. 1 (left), composed of the chemical reactions:

$$
A+B \stackrel{p}{\rightarrow} C, \quad C+D \stackrel{q}{\rightarrow} E+A, \quad E \stackrel{r}{\rightarrow} B+D .
$$

Species $D$ sequestrates $A$ from the complex $C=[A B]$ in order to form the new complex $E=[B D]$, which splits again to release $B$. The associated dynamical system is

$$
\left\{\begin{array}{l}
\dot{a}=-p(a, b)+q(c, d) \\
\dot{b}=-p(a, b)+r(e) \\
\dot{c}=+p(a, b)-q(c, d) \\
\dot{d}=-q(c, d)+r(e) \\
\dot{e}=+q(c, d)-r(e)
\end{array}\right.
$$

where the state vector is $\left[\begin{array}{lllll}a & b & c & d & e\end{array}\right]^{\top}$, the reaction rate vector is $\left[\begin{array}{lll}p(a, b) & q(c, d) & r(e)\end{array}\right]^{\top}$ and the stoichiometric matrix is

$$
S=\left[\begin{array}{ccc}
-1 & 1 & 0 \\
-1 & 0 & 1 \\
1 & -1 & 0 \\
0 & -1 & 1 \\
0 & 1 & -1
\end{array}\right]
$$

Transposing the stoichiometric matrix $S$ yields the dual system with state vector $\left[\begin{array}{lll}p & q & r\end{array}\right]^{\top}$, reaction rate vector $\left[\begin{array}{lllll}a(p) & b(p) & c(q) & d(q) & e(r)\end{array}\right]^{\top}$ (adopting the same letters, with swapped role) and stoichiometric matrix $S^{\top}$

$$
\left\{\begin{array}{l}
\dot{p}=-a(p)-b(p)+c(q) \\
\dot{q}=a(p)-c(q)-d(q)+e(r) \\
\dot{r}=b(p)+d(q)-e(r)
\end{array}\right.
$$

corresponding to the dual $\mathrm{CRN}^{*}$

$$
P \stackrel{a}{\rightarrow} Q, \quad P \stackrel{b}{\rightarrow} R, \quad Q \stackrel{c}{\rightarrow} P, \quad Q \stackrel{d}{\rightarrow} R, \quad R \stackrel{e}{\rightarrow} Q .
$$

The dual network is associated with a structurally stable monomolecular reaction network (where all reactions have the form $X \rightarrow Y$ ): as we will see, this allows us to claim that the primal network is structurally stable too.
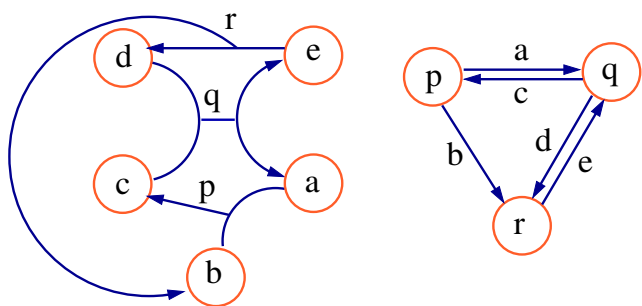

Figure 1: The reaction network in Example 1 (left) and its dual (right).

To write the system in reaction rate coordinates (see [1], [2], [3], [4], as well as [8], and the references therein), we introduce the rate variable $r(t) \doteq g(x(t))$. Recall that $g(\bar{x})=g(0)=0$ in view of Assumption 1. The system of rate equations is achieved by differentiating $\frac{d}{d t} r=\frac{\partial g}{\partial x} \dot{x}=\frac{\partial g}{\partial x} S r$, hence

$$
\dot{r}(t)=\frac{\partial g}{\partial x} \operatorname{Sr}(t)
$$

We can define a system of rate equations for the dual network as well, by considering $w(t) \doteq h(z(t))$, and we get

$$
\dot{w}(t)=\frac{\partial h}{\partial z} S^{\top} w(t)
$$

Due to the possible presence of conservation laws, any chemical reaction network of the form (2) evolves in a positively invariant subspace denoted as stoichiometric compatibility class (SC) [14], [16], [18]:

$$
\mathcal{S}=\operatorname{Ra}[S]=\left\{x: x=S v, \quad v \in \mathbb{R}^{m}\right\},
$$

where $\operatorname{Ra}[S]$ is the range, or image, of matrix $S$. In the original coordinates, the SC for (1) would be an affine manifold, $\tilde{\mathcal{S}}\left(y_{0}\right)=\left\{y \geq 0: y=y_{0}+S v, \quad v \in \mathbb{R}^{m}\right\}$, depending on the initial conditions $y(0)=y_{0}$. Since we assumed that an equilibrium exists for (1), and it is at the origin for the shifted system (2), we can write $\mathcal{S}$ as the linear manifold above. Our structural analysis allows us to assess the stability of the equilibrium regardless of the original (pre-translation) stoichiometric compatibility class and equilibrium value; this confers an intrinsic robustness with respect to the ubiquitous noise one encounters in experimental settings.

Definition 2: A system of the form (2) is

- stable in the stoichiometric compatibility class (SSC) if, for every initial condition of the form $x(0)=S v_{0}$, with $v_{0} \in \mathbb{R}^{m}$, the state $x(t)$ converges to 0 as $t \rightarrow \infty$;

- locally stable in the stoichiometric compatibility class (LSSC) if the linearised system at 0 ,

$$
\dot{x}=S\left[\frac{\partial g}{\partial x}(\bar{x})\right]_{\bar{x}=0} x,
$$

is stable in the stoichiometric compatibility class (which is the same as for the nonlinear system).

The class of systems of the form (2) is structurally stable, or locally stable, in the stoichiometric compatibility class (resp. 
S-SSC and S-LSSC) if the above conditions hold for arbitrary vector functions $g$ satisfying our assumptions.

We now observe that an interesting relation exists between the structure of a system and the structure of its dual.

To this aim, we define the qualitative class $\Sigma$ of nonnegative matrices that have positive entries corresponding to the negative entries of the stoichiometric matrix $S$ and zero entries elsewhere:

$$
\Sigma=\{M: \operatorname{sign}(M)=\operatorname{sign}(\max \{-S, 0\})\} .
$$

We also define $\Sigma^{\top}=\left\{M: M^{\top} \in \Sigma\right\}$.

For instance, for the class of systems (4) in Example 1,

$$
\Sigma=\left\{M: \operatorname{sign}(M)=\left[\begin{array}{rrr}
+1 & 0 & 0 \\
+1 & 0 & 0 \\
0 & +1 & 0 \\
0 & +1 & 0 \\
0 & 0 & +1
\end{array}\right]\right\} .
$$

Let us denote $\frac{\partial g}{\partial x} \doteq G$ and $\frac{\partial h}{\partial z} \doteq H$. We notice that $G \in \Sigma^{\top}$, because the non-zero partial derivatives of $g$, which are positive in view of Assumption 4, are associated with the negative entries of $S$ and correspond to the negative entries of $S^{\top}$ as per Assumption 3 ( $g_{j}$ depends on $x_{i}$ if and only if $S_{i j}<0$ ). Analogously, $H \in \Sigma$, because $h_{j}$ depends on $z_{i}$ if and only if $S_{i j}^{\top}<0$, and all its non-zero partial derivatives are positive.

Then, we can write the Jacobian of system (2) as

$$
S \frac{\partial g}{\partial x} \doteq S G, \quad \text { with } G \in \Sigma^{\top},
$$

and the Jacobian of the dual system (3) as

$$
S^{\top} \frac{\partial h}{\partial z} \doteq S^{\top} H, \quad \text { with } H \in \Sigma .
$$

\section{Structural Local Stability Analysis}

We prove our main result concerning structural local stability in the stoichiometric compatibility class.

Theorem 1: System (2) is S-LSSC if and only if its dual system (3) is S-LSSC.

Proof: First, notice that (2) is S-LSSC if and only if $S G$ is Hurwitz in the stoichiometric compatibility class (HSC; namely, any trajectory originated in the SC converges to zero) for all $G \in \Sigma^{\top}$. Indeed, given some matrix $G \in \Sigma^{\top}$, we can always find functions $g_{k}$, possibly linear, whose derivatives at zero are equal to the entries of $G$ (cf. also [9], where a similar construction is proposed). We therefore wish to show that $S G$ is HSC for all $G \in \Sigma^{\top}$ if and only if $S^{\top} H$ is HSC for all $H \in \Sigma$. Consider $\operatorname{rank}[S]=r \leq n$ and apply a similarity transformation $T$ such that

$$
\begin{aligned}
T_{S G T^{-1}} & =\left[\begin{array}{cc}
\tilde{S}_{1} & \tilde{S}_{2} \\
0 & 0
\end{array}\right]\left[\begin{array}{cc}
\tilde{G}_{1} & \tilde{G}_{2} \\
\tilde{G}_{3} & \tilde{G}_{4}
\end{array}\right] \\
=M_{1} & =\left[\begin{array}{cc}
\tilde{S}_{1} \tilde{G}_{1}+\tilde{S}_{2} \tilde{G}_{3} & \tilde{S}_{1} \tilde{G}_{2}+\tilde{S}_{2} \tilde{G}_{4} \\
0 & 0
\end{array}\right],
\end{aligned}
$$

where $\tilde{S}_{1}$ and $\tilde{S}_{2}$ have $r$ rows. In the new representation, the initial conditions in the $\mathrm{SC}$ are all vectors of the form $\left[x_{1}^{\top} \mathbf{0}\right]^{\top}$, with $x_{1} \in \mathbb{R}^{r}$ and $\mathbf{0}$ a row vector of $n-r$ zeros. Hurwitz stability in the SC is equivalent to $x_{1}(t) \rightarrow 0$ for each initial vector $\left[x_{1}^{\top}(0) \quad \mathbf{0}\right]^{\top}$. Structural local stability in the SC is then equivalent to matrix $\tilde{S}_{1} \tilde{G}_{1}+\tilde{S}_{2} \tilde{G}_{3}$ being Hurwitz for all $G \in$ $\Sigma^{\top}$.
The same arguments for $S^{\top} H$ lead to a partition

$$
M_{2}=\left[\begin{array}{cc}
\hat{S}_{1} \hat{H}_{1}+\hat{S}_{2} \hat{H}_{3} & \hat{S}_{1} \hat{H}_{2}+\hat{S}_{2} \hat{H}_{4} \\
0 & 0
\end{array}\right]
$$

and structural local stability in the $\mathrm{SC}$ is equivalent to matrix $\hat{S}_{1} \hat{H}_{1}+\hat{S}_{2} \hat{H}_{3}$ being Hurwitz for all $H \in \Sigma$. Since the two upper-left blocks of $M_{1}$ and $M_{2}$ have size $r$, both matrices have $n-r$ zero eigenvalues.

Matrices $M_{1}$ and $M_{2}$ have the same eigenvalues as $S G$ and $S^{\top} H$, respectively, or equivalently the same eigenvalues as $S G$ and $H^{\top} S$ (by transposition). Matrix $S G$ (respectively $S^{\top} H$ ) is structurally Hurwitz in the SC if and only if, for every $G \in \Sigma^{\top}$ (respectively $H \in \Sigma$ ), it has precisely $n-r$ zero eigenvalues and $r$ eigenvalues with negative real part.

Since $G$ and $H^{\top}$ have the same structure $\left(G, H^{\top} \in \Sigma^{\top}\right)$, we can always pick $H=G^{\top}$. The proof is concluded by recalling that $S G$ and $H^{\top} S=G S$ share the same non-zero eigenvalues (just take $G S x=\lambda x \neq 0$, hence $S G(S x)=$ $\lambda(S x) \neq 0)$, which have a negative real part.

The structural local stability of the primal/dual class of systems can be assessed via convex optimisation, parametric Lyapunov functions or deficiency theory [12], [14], [16].

Example 2: Consider the CRN in Example 1. Its dual $\mathrm{CRN}^{*}$ is mono-molecular and has a weakly diagonally dominant Jacobian, hence structural local stability in the SC is guaranteed for both the dual and the primal system.

\section{JaCobian DeCOMPosition AND DUALITY}

We recall that the Jacobian of system (2) can always be decomposed as [7], [9]

$$
S G=B \Delta C,
$$

for all $G \in \Sigma^{\top}$, where the diagonal matrix $\Delta$, of size $q$, carries on the diagonal all the non-zero entries of $G$. To generate the constant matrices $B$ and $C$, the $q$ non-zero partial derivatives need to be ordered as $\Delta_{k}=\partial g_{j} / \partial x_{i}=G_{i j}>0, k=1, \ldots q$, so that two indices $i(k)$ and $j(k)$ correspond to each $k$. Then, for each $k$ :

- the $k$ th column of $B$ is the $j$ th column of $S, j=j(k)$,

- the $k$ th row of $C$ is $e_{i}^{\top}, i=i(k)$,

where $e_{i}^{\top}$ is the $i$ th row vector of the canonical basis. The pair $(B, C)$ captures the system structure, and a given choice of the diagonal entries of $\Delta$ yields a specific realisation.

The same property holds for the rate representation [8]. We can associate the linearisation of (6) with matrix

$$
G S=E \Delta F,
$$

where the constant matrices $E$ and $F$ (after ordering the nonzero partial derivatives $\left.\Delta_{k}=\partial g_{j} / \partial x_{i}=G_{i j}>0\right)$ are formed as follows:

- the $k$ th column of $E$ is $e_{j}, j=j(k)$,

- the $k$ th row of $F$ is $S_{i}^{\top}$, the $i$ th row of $S, i=i(k)$, where $e_{j}$ is the $j$ th column vector of the canonical basis. Now the system structure is captured by the pair $(E, F)$.

In the sequel we assume that the order of the partial derivatives in $\Delta$ is the same for both representations (in concentrations and in rates). 
Example 3: Consider the CRN associated with equations

$$
\left\{\begin{array}{l}
\dot{a}=-g_{a}(a)-g_{a c}(a, c) \\
\dot{b}=g_{a}(a)-g_{b c}(b, c) \\
\dot{c}=g_{a}(a)-g_{a c}(a, c)-g_{b c}(b, c)
\end{array}\right.
$$

corresponding to the general model (2) with $x=\left[\begin{array}{lll}a & b & c\end{array}\right]^{\top}$,

$$
S=\left[\begin{array}{ccc}
-1 & -1 & 0 \\
1 & 0 & -1 \\
1 & -1 & -1
\end{array}\right], \quad g(x)=\left[\begin{array}{c}
g_{a}(a) \\
g_{a c}(a, c) \\
g_{b c}(b, c)
\end{array}\right] \text {. }
$$

By ordering the partial derivatives as $\Delta_{1} \doteq \partial g_{a} / \partial a, \Delta_{2} \doteq$ $\partial g_{a c} / \partial a, \Delta_{3} \doteq \partial g_{a c} / \partial c, \Delta_{4} \doteq \partial g_{b c} / \partial b, \Delta_{5} \doteq \partial g_{b c} / \partial c$, the system structure is captured by the $B \Delta C$ decomposition with matrices

$$
B=\left[\begin{array}{rrrrr}
-1 & -1 & -1 & 0 & 0 \\
1 & 0 & 0 & -1 & -1 \\
1 & -1 & -1 & -1 & -1
\end{array}\right] \quad \text { and } \quad C=\left[\begin{array}{lll}
1 & 0 & 0 \\
1 & 0 & 0 \\
0 & 0 & 1 \\
0 & 1 & 0 \\
0 & 0 & 1
\end{array}\right] \text {, }
$$

while the $E \Delta F$ decomposition has matrices

$$
E=\left[\begin{array}{lllll}
1 & 0 & 0 & 0 & 0 \\
0 & 1 & 1 & 0 & 0 \\
0 & 0 & 0 & 1 & 1
\end{array}\right] \text { and } F=\left[\begin{array}{rrr}
-1 & -1 & 0 \\
-1 & -1 & 0 \\
1 & -1 & -1 \\
1 & 0 & -1 \\
1 & -1 & -1
\end{array}\right] \text {. }
$$

The dual chemical reaction network $\mathrm{CRN}^{*}$ admits the same types of decomposition, $B^{*} \Delta^{*} C^{*}$ and $E^{*} \Delta^{*} F^{*}$, in concentrations and in rates.

Numerically, the partial derivatives of a system and of its dual could be different. However, the numerical values of the parameters do not affect our parameter-free analysis, which is focused on the structure: in the sequel, the statement $J=B \Delta C$ means that the structure of the system Jacobian is captured by the matrix pair $(B, C)$, namely that the equality holds for some (no matter which) $\Delta \in \mathcal{D}$, where $\mathcal{D}$ is the family of diagonal matrices, of size $q$, with positive diagonal entries. Hence, in the following we will drop the star in $\Delta^{*}$ and denote by $\Delta$ the generic element of the family $\mathcal{D}$. Then, the dual decomposition is related to the primal decomposition as shown next (see Table I).

Theorem 2: Consider the CRN system (2) and its dual $\mathrm{CRN}^{*}$ system (3), along with their Jacobian decompositions with the non-zero partial derivatives ordered in a diagonal matrix $\Delta \in \mathcal{D}$. Then:

- the dual $\mathrm{CRN}^{*}$ in concentrations has the same structure as the transpose of the primal CRN in rates:

$$
B^{*} \Delta C^{*}=F^{\top} \Delta E^{\top}, \quad \Delta \in \mathcal{D} ;
$$

- the dual $\mathrm{CRN}^{*}$ in rates has the same structure as the transpose of the primal CRN in concentrations:

$$
E^{*} \Delta F^{*}=C^{\top} \Delta B^{\top}, \quad \Delta \in \mathcal{D} .
$$

Proof: The primal CRN has Jacobian $S G=B \Delta C$ for some $G \in \Sigma^{\top}$, while the dual $\mathrm{CRN}^{*}$ has Jacobian $S^{\top} H=$ $B^{*} \Delta C^{*}$ for some $H \in \Sigma$. For the primal rate representation, $G S=E \Delta F$ for some $G \in \Sigma^{\top}$, hence $S^{\top} G^{\top}=F^{\top} \Delta E^{\top}$ for some $G^{\top} \in \Sigma$. This proves the first statement, since $H, G^{\top} \in$ $\Sigma$ have the same structure. Also, for the dual system in rates, $H S^{\top}=E^{*} \Delta F^{*}$ for some $H \in \Sigma$. Since $G^{\top} S^{\top}=C^{\top} \Delta B^{\top}$ for some $G^{\top} \in \Sigma$, the second statement is also proven.
Table I: The duality (transpose) relation among Jacobian representations.

\begin{tabular}{|l|c|c|}
\hline & in concentrations: & in rates: \\
\hline $\mathrm{CRN}:$ & $B \Delta C$ & $E \Delta F$ \\
\hline $\mathrm{CRN}^{*}:$ & $F^{\top} \Delta E^{\top}$ & $C^{\top} \Delta B^{\top}$ \\
\hline
\end{tabular}

\section{LyapunOV NORMS AND DUALITy FOR GLOBAL STABILITY ANALYSIS}

To derive a global stability condition, we first examine the relation between the representations in concentrations and in rates. Since $g(0)=0$ in view of Assumption 1, if $x(t) \rightarrow 0$, then also $r(t)=g(x(t))$ converges to zero. Conversely, if we assume that $S g(\bar{x})=0$ implies $\bar{x}=0$ (i.e., zero is the only equilibrium in the SC), then convergence of $r=g(x)$ to zero implies $x(t) \rightarrow 0$.

Structural convergence of $x(t)$ to zero can be proven by absorbing system (2) in a differential inclusion. Indeed, the same matrices $(B, C)$ as in the Jacobian decomposition (11) can be used to provide a global representation of the system. Consider the general property

$$
S g(x)=S g(\bar{x})+\left[\int_{0}^{1} J(\bar{x}+\sigma(x-\bar{x})) d \sigma\right](x-\bar{x}),
$$

where $J=\frac{\partial}{\partial x}[S g(x)]$. Since $J=B \Delta C$ in view of (11),

$$
\int_{0}^{1} J d \sigma=B\left(\int_{0}^{1} \Delta d \sigma\right) C=B D(x) C
$$

for some diagonal $D(x)$ (see [9], [10] for details). We also define $\hat{G}(x) \doteq \int_{0}^{1} \frac{\partial g}{\partial x}(\bar{x}+\sigma(x-\bar{x})) d \sigma$. Since $\bar{x}=0$, system (2) admits the equivalent global representation

$$
\dot{x}(t)=S \hat{G}(x(t)) x(t)=B D(x(t)) C x(t),
$$

where matrix $D$ depends on the current state $x(t)$. For our structural analysis, we drop the dependence of $D$ on $x$ to consider a generic diagonal matrix $D(t)$ with positive diagonal entries. The set of solutions of system (2) is always a subset of the solutions of the differential inclusion [7], [9]

$$
\dot{x}(t)=S \hat{G}(t) x(t), \quad \hat{G}(t) \in \Sigma^{\top},
$$

or equivalently

$$
\dot{x}(t)=B D(t) C x(t), \quad D(t) \in \mathcal{D} .
$$

Therefore, the stability of the differential inclusion implies the structural stability of (2); note that systems (14) and (15) have the same stoichiometric compatibility class, because matrices $S$ and $B$ have the same range.

We can analogously prove the convergence in rate coordinates [1], [2], [3], [4], [8], since all the solutions of system (6) are a subset of the solutions of

$$
\dot{r}(t)=G(t) \operatorname{Sr}(t), \quad G(t) \in \Sigma^{\top},
$$

(again we drop the dependence of $G=\partial g / \partial x$ on $x$ ), or equivalently

$$
\dot{r}(t)=E D(t) F r(t), \quad D(t) \in \mathcal{D} .
$$


Table II: The four system representations.

\begin{tabular}{|l|c|c|}
\hline & in concentrations: & in rates: \\
\hline $\mathrm{CRN}:$ & $\dot{x}(t)=B D(t) C x(t)$ & $\dot{r}(t)=E D(t) F r(t)$ \\
\hline $\mathrm{CRN}^{*}:$ & $\dot{z}(t)=F^{\top} D(t) E^{\top} z(t)$ & $\dot{w}(t)=C^{\top} D(t) B^{\top} w(t)$ \\
\hline
\end{tabular}

Stability of system (17) implies convergence to zero of the rate variable $r(t)$.

Analogous representations in concentrations and in rates hold for the dual $\mathrm{CRN}^{*}$. The four representations are summarised in Table II.

We now consider Lyapunov norms, according to the following definition.

Definition 3: A norm $V(x)$ is a Lyapunov norm if it is nonincreasing along the trajectories of system (15) or (17). If $V(x)$ is polyhedral, we call it Polyhedral Lyapunov Function (PLF). $\diamond$

Remark 2: The existence of a Lyapunov norm is not enough to ensure asymptotic stability. We also need an assumption on the partial derivatives: there exist two (unknown) numbers $\epsilon$, no matter how small, and $\mu$, no matter how large, such that

$$
0<\epsilon \leq D_{i}(t) \leq \mu
$$

Without this assumption, no robust convergence can be claimed: for instance, $\dot{x}=-d(t) x(t)$ does not converge to zero for all $d(t)>0$, even though $|x(t)|$ is decreasing. Conversely, provided that (18) holds, if the Lyapunov norm is polyhedral and the differential inclusion is non-singular, then asymptotic stability is ensured [9].

Given a norm $V(x)$, its dual is defined as

$$
V^{*}(z)=\max _{V(x) \leq 1} x^{\top} z
$$

Given a full-row-rank matrix $X$, a polyhedral norm can be defined through a vertex representation as

$$
V_{X}(x)=\inf \left\{\|p\|_{1}: X p=x\right\},
$$

while, given a full-column-rank matrix $Z$, a polyhedral norm can be defined through a plane representation as

$$
U_{Z}(z)=\|Z z\|_{\infty} .
$$

As shown in [11, Proposition 4.35], a duality relation exists between (19) and (20):

$$
V_{X}^{*}(x)=U_{Z}(z) \text { if } Z=X^{\top} .
$$

Theorem 3: Consider the four representations in Table II. The CRN in concentrations ( $x$-system) admits a PLF if and only if the $\mathrm{CRN}^{*}$ in rates ( $w$-system) admits the dual PLF; the same holds for the $z$-system and the $r$-system.

Proof: Given a polyhedral norm $V$ and a matrix $A$, we define the matrix measure as

$$
\mu_{V}(A)=\lim _{h \rightarrow 0} \frac{V(I+h A)-1}{h},
$$

where $V(M)$ is the induced norm of matrix $M$. Since $V(M)=V^{*}\left(M^{\top}\right)$, we have $\mu_{V}(A)=\mu_{V^{*}}\left(A^{\top}\right)$. Assume that (15) admits a PLF $V(x)$ or, equivalently, that $V(x)$ is a common PLF for all linear systems with matrix $B D C, D \in \mathcal{D}$. Consider expression (19) for $V$; the proof can be also carried out using (20). Then, for any $D \in \mathcal{D}$ we must have

$$
B D C X=X P(D)
$$

with $\mu_{V_{1}}(P(D)) \leq 0$ [11]. Transposing both sides yields

$$
X^{\top} C^{\top} D B^{\top}=P(D)^{\top} X^{\top}
$$

with $\mu_{V_{\infty}}\left(P(D)^{\top}\right) \leq 0$. This implies that the dual norm $V^{*}(x)$ is a PLF for all linear systems with transposed matrix $C^{\top} D B^{\top}, D \in \mathcal{D}$ (see again [11] for details).

Remark 3: Theorem 3 answers the question: which are the most suitable polyhedral Lyapunov functions to prove the structural stability of chemical reaction networks, those in concentrations or those in rates? The answer is a draw: for any network admitting a PLF in concentrations, there is another network, the dual, admitting a PLF in rates; and vice versa. $\diamond$

Polyhedral functions are amenable for efficient computation [7], [4]. Moreover, it has been shown that there are examples of networks for which the only possible structural Lyapunov function is polyhedral [9]; Theorem 3 implies that there are networks for which piecewise-linear functions in rates [1], [2], [3], [4] have the same exclusive property.

Remark 4: The result can be generalised to any norm [17]: if $V$ is a Lyapunov norm for (15), then $V^{*}$ is a Lyapunov norm for the rate representation of the dual.

\section{APPLICATIONS AND CONCLUDING DisCUSSION}

We discuss in this section some implications of our duality results and their applicability to gain additional insight into chemical reaction networks.

First, duality can be exploited to immediately assess the structural stability of a complex chemical reaction network by simply inspecting its topology. Let us consider unitary networks, namely CRNs such that all entries $S_{i j} \in\{-1,0,1\}$. The next result is proven in [7].

Proposition 1: Assume that, in a unitary CRN, each reaction affects at most two nodes (species), i.e. there are at most two non-zero coefficients in each column of $S$. Then, the network is structurally stable with $\|x\|_{1}$ as a PLF.

The conditions in Proposition 1 are extremely simple to check, even for very large CRNs. The same is true for the conditions in the following new result, which we can prove by adopting duality.

Proposition 2: Assume that, in a unitary CRN, each node is affected by at most two reactions, i.e. there are at most two non-zero coefficients in each row of $S$. Then, the network is structurally stable with $\|w\|_{\infty}$ as a PLF in rates.

Proof: It follows from Proposition 1 and Theorem 3, since $V_{\infty}=\|\cdot\|_{\infty}$ is the dual norm of $V_{1}=\|\cdot\|_{1}$.

For instance, it can be immediately seen from Fig. 1 (left) that the CRN in Example 1 satisfies the conditions of Proposition 2, hence it is structurally stable in the SC. 
Example 4: The metabolic network

$$
\left\{\begin{array}{l}
\dot{a}=-p(a, e) \\
\dot{b}=p(a, e)-q(b) \\
\dot{c}=q(b)-r(f, c) \\
\dot{d}=r(f, c)-s(d) \\
\dot{e}=-p(a, e)+r(f, c) \\
\dot{f}=-r(f, c)+p(a, e)
\end{array}\right.
$$

adapted from [22, Chapter 17] has the form (2) with

$$
S=\left[\begin{array}{rrrr}
-1 & 0 & 0 & 0 \\
1 & 0 & 0 & -1 \\
0 & -1 & 0 & 1 \\
0 & 1 & -1 & 0 \\
-1 & 1 & 0 & 0 \\
1 & -1 & 0 & 0
\end{array}\right]
$$

It can be seen that the $\mathrm{SC}$ is identified by the conservation laws $b+c+e=$ constant and $e+f=$ constant, and an equilibrium exists at zero. The dual network

$$
\left\{\begin{array}{l}
\dot{p}=-a(p)+b(q)-e(p)+f(r) \\
\dot{r}=-c(r)+d(s)+e(p)-f(r) \\
\dot{s}=-d(s) \\
\dot{q}=-b(q)+c(r)
\end{array}\right.
$$

is mono-molecular and satisfies the assumptions of Proposition 2 (there are at most two non-zero entries in each row of $S$ ), hence also the primal network is structurally stable.

It is interesting to observe that the dual of a reversible network (such that all reactions are reversible) is not reversible, and not even weakly reversible according to the definition in [16]. If the reaction rates are of the mass-action type, i.e. of the form $\kappa a^{m} b^{n}$, these functional expressions are not preserved for the dual.

Example 5: The dual of the reversible network

$$
2 A+B \underset{q}{\stackrel{p}{\rightleftharpoons}} C \underset{s}{\stackrel{r}{\rightleftharpoons}} D
$$

is the non-reversible network

$$
2 P \stackrel{a}{\rightarrow} 2 Q, \quad P \stackrel{b}{\rightarrow} Q, \quad Q+R \stackrel{c}{\rightarrow} P+S, \quad S \stackrel{d}{\rightarrow} R .
$$

Under mass-action kinetics, the primal has reaction rate functions $p=\alpha a^{2} b, q=\beta c, r=\gamma c, s=\delta d$, while the dual has reaction rate functions $a=\epsilon p^{2}, b=\eta p, c=\mu q r, d=\nu s$. $\diamond$

Very interestingly, duality results can enable us to easily assess the structural stability of non-unitary networks, for which computing PLFs is not easy [7].

Example 6: The reversible network in Example 5 is nonunitary. To study its structural stability in the SC, we can consider its dual network

$$
\left\{\begin{array}{l}
\dot{p}=-2 a(p)-b(p)+c(q, r)=-g(p)+c(q, r) \\
\dot{q}=+2 a(p)+b(p)-c(q, r)=g(p)-c(q, r) \\
\dot{r}=-c(q, r)+d(s) \\
\dot{s}=+c(q, r)-d(s)
\end{array}\right.
$$

where $g(p) \doteq 2 a(p)+b(p)$. This new network with $g(p)$ is unitary and satisfies Proposition 2, hence the system is structurally stable, because it admits a piecewise-linear in rates Lyapunov function.
Future research directions are aimed at investigating how the relation between primal and dual chemical reaction networks reflects on the structure of other types of graph representations, such as the Species-Reaction Graph [16], and at exploiting dual networks to assess properties other than stability.

\section{REFERENCES}

[1] M. A. Al-Radhawi and D. Angeli, "Piecewise linear in rates Lyapunov functions for complex reaction networks," in 52rd IEEE Conference on Decision and Control, 2013, pp. 4595-4600.

[2] M. A. Al-Radhawi and D. Angeli, "Robust Lyapunov functions for complex reaction networks: An uncertain system framework," in 53rd IEEE Conference on Decision and Control, 2014, pp. 3101-3106.

[3] M. A. Al-Radhawi and D. Angeli, "New approach to the stability of chemical reaction networks: Piecewise linear in rates Lyapunov functions," IEEE Trans. Autom. Control, 61(1), 76-89, 2016.

[4] M. A. Al-Radhawi, D. Angeli and E. D. Sontag, "A computational framework for a Lyapunov-enabled analysis of biochemical reaction networks", PLOS Computational Biology, 16(2), 2020.

[5] D. Anderson, "Global asymptotic stability for a class of nonlinear chemical equations," SIAM Journal on Applied Mathematics, 68(5), 1464-1476, 2008.

[6] D. Angeli, "A tutorial on chemical reaction network dynamics", European J. Control, 15(3-4), 398-406, 2009.

[7] F. Blanchini and G. Giordano, "Piecewise-linear Lyapunov functions for structural stability of biochemical networks," Automatica, 50(10), 2482-2493, 2014.

[8] F. Blanchini and G. Giordano, "Polyhedral Lyapunov functions for structural stability of biochemical systems in concentration and reaction coordinates", 54th IEEE Conference on Decision and Control, pp. 3110$3115,2015$.

[9] F. Blanchini and G. Giordano, "Polyhedral Lyapunov functions structurally ensure global asymptotic stability of dynamical networks iff the Jacobian is non-singular", Automatica, 86(12), 183-191, 2017.

[10] F. Blanchini and G. Giordano, "Structural analysis in biology: a control theoretic approach", Automatica, 126, 109376, 2021.

[11] F. Blanchini and S. Miani, Set-theoretic methods in control, Systems and Control: Foundations and Applications, pp. 1-630, 2015.

[12] F. Blanchini, G. Chesi, P. Colaneri and G. Giordano, "Checking structural stability of BDC-decomposable systems via convex optimisation", IEEE Control Systems Letters, 4(1), 205-210, 2020.

[13] M. Chaves, "Stability of rate-controlled zero-deficiency networks," in 45th IEEE Conference on Decision and Control, 2006, pp. 5766-5771.

[14] B. L. Clarke, "Stability of Complex Reaction Networks", in Advances in Chemical Physics (eds I. Prigogine \& S. A. Rice), 1980.

[15] I. Famili and B. O. Palsson, "The convex basis of the left null space of the stoichiometric matrix leads to the definition of metabolically meaningful pools", Biophysical Journal, 85, 16-26, 2003.

[16] M. Feinberg, Foundation of Chemical Reaction Network Theory, Springer, 2019

[17] R. Goebel, A. R. Teel, T. Hu and Z. Lin, "Conjugate convex Lyapunov functions for dual linear differential inclusions," IEEE Trans. Autom. Control, 51(4), 661-666, 2006.

[18] F. Horn and R. Jackson, "General mass action kinetics," Archive for Rational Mechanics and Analysis, 47(2), 81-116, 1972.

[19] F. Horn, "On a connexion between stability and graphs in chemical kinetics. I. stability and the reaction diagram," Royal Society of London Proceedings Series A, 334, 299-312, 1973.

[20] F. Horn, "On a connexion between stability and graphs in chemical kinetics. II. stability and the complex graph," Royal Society of London Proceedings Series A, 334, 313-330, 1973.

[21] E. D. Sontag, "Structure and stability of certain chemical networks and applications to the kinetic proofreading model of T-Cell receptor signal transduction," IEEE Trans. Autom. Control, 46, 1028-1047, 2001.

[22] D. Voet and J. Voet, Biochemistry, John Wiley and sons, 2011. 